\title{
On the Nature of Large Scale Wave Disturbances in the Equatorial Lower Stratosphere
}

\author{
By Richard S. Lindzen* \\ Department of Geophysical Sciences, University of Chicago \\ and Taroh Matsuno \\ Department of Physics, Kyushu University, Fukuoka \\ (Manuscript received 24 November 1967, in revised form 20 April 1969)
}

\begin{abstract}
Large scale wave disturbances in equatorial regions, and, in particular, those described by Maruyama (1967), are discussed in terms of forced oscillations. In doing this we are able to explain the importance of the waves' tilts, the role of the beta effect, and the waves' contributions to the mean zonal momentum, Consideration of the last items leads us to conjecture on the nature of those disturbances in the equatorial stratosphere which were not isolated in the analysis of Maruyama.
\end{abstract}

\section{Introduction}

In analyses of the winds in the lower stratosphere over the equatorial Pacific, planetary scale easterly waves have been found (Yanai and Maruyama, 1966; Maruyama and Yanai, 1967; Maruyama, 1967). The observed waves appear to be associated with a zonal wavenumber of about 4 and a phase speed of about $-23 \mathrm{~m} / \mathrm{s}$ against a mean zonal flow of $+9 \mathrm{~m} / \mathrm{s}$. From these numbers a doppler shifted period of about 4 days may be deduced. The waves are, moreover, associated with a vertical wavelength of about $6 \mathrm{~km}$ and a westward tilt; i.e., the waves' phases are propagated downward.

Maruyama (1967) performed a brief theoretical analysis of these waves-comparing their zonal phase speeds with the predictions of Rossby (1939), Haurwitz (1940), and Rosenthal (1965) and Matsuno (1966). In the predictions of Rosenthal and Matsuno, account was taken of the waves' short vertical wavelengths and modest agreement with observations was obtained. However, all the above analyses (wherein horizontal and vertical structure are assumed and zonal phase speed is computed) are designed for free oscillations, and free oscillations have no tilt (Lind-

* Present affiliation: National Center for Atmospheric Research, Boulder, Colorado. zen (1967 a) and many others). Waves with tilt must be forced, and for forced oscillations it is more reasonable to assume that horizontal structure and phase speed are known, and to solve for the vertical structure of wave as it leaves (if it does leave) the region of excitation. Thus, although Maruyama's analysis is formally correct,** it will prove useful to reanalyze the waves as forced oscillations. In doing so we shall be able to better discuss the waves' tilt and their role in the mean momentum balance. We will also be able to reassess the importance of the waves' horizontal phase speeds and the role of the $\beta$-effect. Finally, we hope to provide some insight into the problem, posed by Maruyama (1967), of the nature of other disturbances in the equatorial stratosphere.

A treatment of forced planetary waves is given in Lindzen (1967a). One aim of that analysis was to determine what happens as we approach the equator to planetary waves of scales such that they would be trapped (with respect to vertical propagation) at midlatitudes according to the analysis of Charney and Drazin (1961). It was found that as one gets sufficiently near the equator waves of

** It should be noted, however, that vertical wavelength is more correctly defined in terms of length rather than pressure difference. The latter is only of local relevence; the former applies to the whole atmosphere. 
any period and zonal wavenumber will become "untrapped" and propagate upward as long period internal gravity-type waves. In section 2 of the present paper we will review, extend and interpret relevant parts of Lindzen (1967 a). In section 3 we will reanalyze the observed waves of Yanai and Maruyama as forced oscillations, and in section 4 we will discuss some implications of our results.

\section{Theoretical review}

In Lindzen (1967 a) the problem of linearized perturbations in an infinite, compressible, hydrostatic, iso-thermal atmosphere on an equatorial beta-plane was considered. The equations, it was found, could be reduced to a single equation in $v^{\prime}$ (the southerly velocity, $v$, times the square root of the basic density, $\left.\rho_{0}\right)$. This, and some subsequent results, are analogous to Matsuno's (1966) results for a barotropic fluid layer. Away from any excitations this equation had solutions of the form

$$
v_{n}^{\prime}=e^{i(\mu z+k x+\hat{\omega} t)} e^{-\frac{1}{2} \xi^{2}} H_{n}(\xi)
$$

where $x=$ distance in west-east direction,

$y=$ distance north of equator,

$z=$ altitude,

$t=$ time,

$\mu=$ vertical wavenumber,

$k=$ west-east wavenumber,

$\hat{\omega}=$ doppler shifted frequency $(\omega+k U$, where $U$ is mean zonal flow),

$n=$ measure of latitude wavenumber, $\xi=\left(\left(\frac{2 \Omega}{a}\right)^{2} \frac{1}{g h}\right)^{1 / 4} y$,

$H_{n}(\xi)=n$th Hermite polynomial in $\xi$, $\beta=2 \Omega / a$

$\Omega=2 \pi / 1$ day,

$a=$ earth's radius,

$h=$ equivalent depth,

$g=$ acceleration of gravity,

$h$, the eigenvalue of the problem, has to satisfy the relation

$$
\begin{aligned}
\sqrt{g h_{n}}= & \frac{a \Omega(2 n+1)}{s^{2}((2 \Omega / s \hat{\omega})-1)} \\
& \times\left[1 \pm\left(1-\left(\frac{\hat{\omega}}{\Omega}\right)^{2}\left(\frac{s}{2 n+1}\right)^{2}\left(\frac{2 \Omega}{s \hat{\omega}}-1\right)\right)^{1 / 2}\right]
\end{aligned}
$$

where

$$
s=a k=0, \pm 1, \pm 2 \cdots \cdots
$$

Assuming $\hat{\omega}>0$, positive values of $s$ correspond to easterly waves, negative values to westerly waves (relative to some mean zonal current, $U$. ) Given $\hat{\omega}, s$, and $n$, (2) determines $h$ and $h$, in turn determines $\mu$ :

$$
\mu= \pm \sqrt{\frac{\kappa}{H h}-\frac{1}{4 H^{2}}}
$$

where $\quad \kappa=(\gamma-1) / \gamma$

$\gamma=C_{p} / C_{v}=1.4$ for air,

$H=\frac{R T_{0}}{g}=$ Atmosphere's scale height,

$R=$ gas constant for air

The nature of (1) is such that $v_{n}^{\prime}$ decays for

$$
y^{2}>y_{d}^{2}=(2 n+1) \frac{\sqrt{g h}}{(2 \Omega / a)}
$$

It may be shown (from the equations in Lindzen (1967a)) that the other oscillatory fields are given by

$$
\begin{aligned}
u_{n}^{\prime}= & e^{i(\mu z+k x+\hat{\omega} t)}\left(\frac{-i \sqrt{g h} C_{1}^{1 / 4}}{\hat{\omega}}\right) \\
& \times\left\{\frac{n H_{n-1}(\xi)}{1-(k / \hat{\omega}) \sqrt{g h}}+\frac{(1 / 2) H_{n+1}(\xi)}{1+(k / \hat{\omega}) \sqrt{g h}}\right\} \\
& \times e^{-\frac{1}{2} \xi^{2}} \\
w_{n}{ }^{\prime}= & e^{i(\mu z+k x+\hat{\omega} t)}\left(\frac{i h C_{1}{ }^{1 / 4}}{\gamma-1}\right) \\
& \times\left[\mu \gamma H-i\left(1-\frac{\gamma}{2}\right)\right] \\
& \times\left\{\frac{n H_{n-1}(\xi)}{1-(k / \hat{\omega}) \sqrt{g h}}-\frac{(1 / 2) H_{n+1}(\xi)}{1+(k / \hat{\omega}) \sqrt{g h}}\right\} \\
& \times e^{-\frac{1}{2} \xi^{2}}
\end{aligned}
$$

and

$$
\begin{aligned}
\delta p_{n}{ }^{\prime}= & e^{i(\mu z+k x+\hat{\omega} t)}\left(\frac{i g h}{\hat{\omega}} C_{1}^{1 / 4}\right) \\
& \times\left\{\frac{n H_{n-1}(\xi)}{1-(k / \hat{\omega}) \sqrt{g h}}-\frac{(1 / 2) H_{n+1}(\xi)}{1+(k / \hat{\omega}) \sqrt{g h}}\right\} \\
& \times e^{-\frac{1}{2} \xi 2}
\end{aligned}
$$

where $u^{\prime}=\rho_{0}{ }^{1 / 2} u$,

$u=$ westerly velocity,

$w^{\prime}=\rho_{0}^{1 / 2} w$,

$w=$ vertical velocity,

$\delta p^{\prime}=\rho_{0}^{-1 / 2} \delta p$, 


$$
\begin{aligned}
& \delta p=\text { pressure perturbation, } \\
& C_{1}=\left(\frac{2 \Omega}{a}\right)^{2} \frac{1}{g h} .
\end{aligned}
$$

The above discussion does not cover the Kelvin wave for which $v^{\prime}=0$.*

\section{a. Tilt}

Above the region of excitation we expect the energy flow to be upwards. This requires $\overline{w^{\prime} \delta p^{\prime}}>0$, which in turn requires that $w^{\prime}$ be less than $90^{\circ}$ out of phase with $\delta p^{\prime}$. From (7) and (8) we have

$$
w^{\prime}=\frac{\hat{\omega}}{g(\gamma-1)}\left(\mu \gamma H-i\left(1-\frac{\gamma}{2}\right)\right) \delta p^{\prime}
$$

From (9) we see that for $\overline{w^{\prime} \delta p^{\prime}}>0, \mu$ must have the same sign as $\hat{\omega}$; i.e., the wave's phase must propagate downward. This is, of course, a well known result found by Wilkes (1949), Eliassen and Palm (1960) and many others. From it we see that the westward tilt of the waves found by Yanai and Maruyama is merely an indication that they are forced from below.

\section{b. Gravity waves versus Rossby waves.}

In equ (2) there are, for any choice of $\hat{\omega}$, $s$, and $n$, two solutions for $\sqrt{g h}$. In general negative solutions are not permissable because for them $\xi^{2} \propto-y^{2}$, and the fields grow with increasing $y^{2}$. Thus, for $s \hat{0}<0$ (i.e., westerly waves) only the solution of (2) with the minus sign is valid; it is of the nature of an internal gravity wave. For $s \hat{\omega}>0$ (i.e., easterly waves) the positive sign in (2) corresponds to internal Rossby-type waves, while the negative sign again corresponds to internal gravity-type waves. In Lindzen (1967 a) it was shown that for $n>0$, the Rossby-type solutions were usually very bad approximations to solutions on a sphere-resulting, it appears, from the unrealistic growth of $f=\beta y$ with increasing $y$. Much better approximations to these solutions were obtained with a midlatitude beta plane.

In this connection it is interesting to take

* The Kelvin wave is dealt with by Matsuno (1966). Observations of such waves in the atmosphere are described by Wallace and Kousky (1968) and the theoretical analysis of these observations is given in Holton and Lindzen (1968). the limit $\hat{\omega} s / \Omega(2 n+1) \gg 1$. (2) becomes

$$
\begin{aligned}
\sqrt{ } g h & =\frac{a \Omega(2 n+1)}{-s^{2}}\left[1 \pm\left(\left(\frac{\hat{\omega}}{\Omega}\right)^{2}\left(\frac{s}{2 n+1}\right)^{2}\right)^{1 / 2}\right] \\
& \approx \mp \frac{a \hat{\omega}}{s}
\end{aligned}
$$

The-sign in (10), corresponding to the + sign in (2), is clearly invalid. If $s \hat{\omega} / \Omega(2 n+1)$ were $\ll-1$, then the + sign in (10) would be invalid.

From (2) we see that $h$ behaves in a singular manner at $2 \Omega / s \hat{\omega}=1$. Namely if $2 \Omega / s \hat{\omega} \sim 1$, (2) may be approximated by

$$
\begin{aligned}
\sqrt{g h_{n+}} & \simeq \frac{2 a \Omega(2 n+1)}{s^{2}(2 \Omega / s \hat{\omega}-1)} \\
\sqrt{g h_{n-}} & \simeq \frac{1}{2(2 n+1)} \frac{a \hat{\omega}^{2}}{\Omega}
\end{aligned}
$$

Clearly $h_{n+}$ goes to infinity as $\hat{\omega} / 2 \Omega$ approaches $1 / s$, while $h_{n-}$ remains finite. Since the equatorial beta-plane approximation is permissible only when the solution is confined near the equator, or $\sqrt{g h} / a \Omega<1$ (Matsuno, 1966 ; Lindzen, 1967a), the $h_{n}$ root becomes invalid for $\hat{\omega} / 2 \Omega \sim 1 / s$. We should discuss the problem by use of the midlatitude beta plane or on the basis of the Laplace's tidal equation on a sphere. According to such studies (Lindzen, 1967a ; Longuet-Higgins, 1968) the above mentioned behavior of $h$ is roughly correct, i.e., the equivalent depth of a Rossby mode $\left(h_{n+}\right)$ becomes infinite at a certain value of $\hat{\omega} / 2 \Omega$, and for larger values, $h$ turns negative. The critical value of $\hat{\omega} / 2 \Omega$ at which the transition from positive to negative $h$ occurs may be obtained from Haurwitz's solution (1940) for free oscillations of what is effectively an infinitely deep fluid. Namely, $h$ is positive or negative according to whether $\hat{\omega} / 2 \Omega$ is smaller or larger than $s /(s+n)(s+n+1)$. Then for the oscillation of interest to us $(s=4-5, \quad \hat{\omega} / \Omega=1 / 3-1 / 5)$, only Rossby type modes of $n=0$ and $n=1$ may have positive equivalent depths. In contrast to this, gravity modes always have positive $h$ 's whose magnitudes decrease with increasing $n$. It is noteworthy that the lateral extent of these modes, $\left[a \sqrt{g h_{n-}} / \Omega \cdot(2 n+1)\right]^{1 / 2}$ is independent of $n$ (Lindzen, 1967 a).

Now if we assume that there exists a thermal excitation of wavenumber $s$ and 
frequency $\hat{\omega}$, it is necessary to expand the latitude dependence of the excitation in terms of the set of eigenfunctions for $\delta p_{n}^{\prime}$, over all $n$ of the both type of solutions. It is known that the energy density of negative $h$ (and very large positive $h$ ) modes decays with height away from the excitation, while positive $h$ modes describe vertically propagating motions. Therefore the energy shared by gravity modes and possibly by the lowest Rossby mode could be propagated vertically, (they are trapped horizontally), whereas part of energy supplied to most of the Rossby modes will be trapped near the level of excitation, though they are more or less wide spread in the horizontal. The situation is very similar to that in the theory of diurnal tide, discussed by Lindzen (1967 b).

\section{c. $n=0$}

As pointed out by Matsuno (1966) and Longuet-Higgins (1968), the case $n=0$ requires special treatment. In this case, the gravity-type solution to (2) is

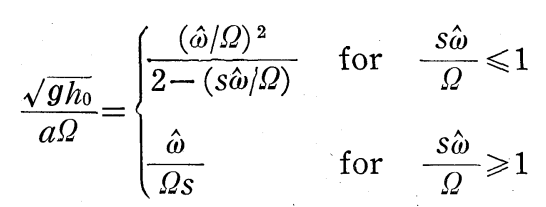

The Rossby-type solution is

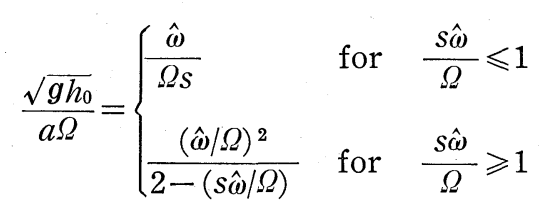

However, as shown by Matsuno (1966) the solution

$$
\frac{\sqrt{g h_{0}}}{a \Omega}=\frac{\hat{\omega}}{\Omega s}
$$

is, in general, invalid. Thus the valid solution is a mixed gravity-Rossby mode, for which

$$
\frac{\sqrt{g h_{0}}}{a \Omega}=\frac{(\hat{\omega} / \Omega)^{2}}{2-(s \hat{\omega} / \Omega)}
$$

Moreover, even this solution cannot exist for $s \hat{\omega} / \Omega>2$; i.e., for $s \hat{\omega} / \Omega>2$ there would appear to be no $n=0$ mode.

\section{d. Momentum transport}

From equations (6) and (8) we see that $u^{\prime}$ and $\delta p^{\prime}$ must either be in phase or $180^{\circ}$ out of phase. Thus, a vertical energy flow (finite $\left.\overline{w^{\prime} \delta p^{\prime}}\right)$ also implies a vertical flux of zonal momentum (finite $\overline{w^{\prime} u^{\prime}}$ ). Consider now the case where (10) (with the correct sign choice) is relevant. Then, for $\hat{\omega} / k$ positive (i.e., easterly waves relative to the mean zonal current, $U), u_{n}{ }^{\prime}$ and $\delta p_{n}{ }^{\prime}$, as given by (6) and (8), will be dominated by the $H_{n-1}(\xi)$ contributions, and they will be $180^{\circ}$ out of phase with each other. Similarly, for $\hat{\omega} / k$ negative (i.e., westerly waves relative to $U$ ), $u_{n}^{\prime}$ and $\delta p_{n}^{\prime}$ ' will be dominated by the $H_{n+1}(\xi)$ contributions, and they will be in phase with each other. Thus, for upward energy flux $\left(\overline{w^{\prime} \delta p^{\prime}}\right.$ $>0$ ), waves easterly relative to $U$ carry easterly momentum upwards and waves westerly relative to $U$ carry westerly momentum upwards. This is merely an expression of the well known relation for pure internal gravity waves. Namely

$$
\overline{w^{\prime} \delta p^{\prime}}=-\rho_{o}(U-c) \overline{u^{\prime} w^{\prime}},
$$

where $c=-\omega / k$ (Eliassen and Palm (1960)). The relation appears to be approximately appropriate for gravity-type waves on the equatorial beta plane as well (Matsuno, 1966).

The $n=0$ mode is again an exception. In this case $H_{n-1}(\xi)$ is identically zero, and both easterly and westerly waves carry westerly momentum.

\section{Waves observed by Yanai and Maruyama}

The waves described by Maruyama (1967) appear to belong to $n=0$ modes. Their doppler shifted period appears to be between 3.5 and 5 days, and their zonal wave number appears to be between 4 and 5 . Using (13) we see that for such waves $h$ should lie between about $0.027 \mathrm{~km}$ and $0.45 \mathrm{~km}$. From (4) we have

$$
L=\frac{2 \pi}{\sqrt{(\kappa / H h)-\left(1 / 4 H^{2}\right)}}
$$

where $L=$ vertical wavelength. Taking $H=6$ $\mathrm{km}\left(T_{0}=205^{\circ} \mathrm{K}\right)$ we find that $L$ lies between about $4.7 \mathrm{~km}$ and $20 \mathrm{~km}$. The observations of Yanai and Maruyama all fall into this range. 
The waves shown in Fig. 15 of Maruyama (1967) appear to have a vertical wavelength of about $6 \mathrm{~km}$. From (15) we estimate that this corresponds to $h \approx 0.043 \mathrm{~km}$. From (1) we have that $v_{0}^{\prime}$ behaves as $\exp -1 / 2 \xi^{2}$ which has half its equatorial value for

$$
\frac{1}{2} \xi^{2}=\frac{1}{2}\left(\frac{2 \Omega}{a}\right) \frac{1}{\sqrt{g} h} y^{2}=0.693
$$

For $h=0.043 \mathrm{~km}$ this corresponds to $1 y 1 \approx 1.12$ $\times 10^{3} \mathrm{~km}$-again in agreement with the waves described by Yanai and Maruyama. Assuming an amplitude for $v$ of $2 \mathrm{~m} / \mathrm{s}$ at the equator, (7) may be used to calculate an amplitude of about $0.08 \mathrm{~cm} / \mathrm{s}$ for $w$. This is somewhat larger than Maruyama's estimate, but given various uncertainties, the agreement is fair.*

In view of the above agreement, it appears that the identification of the waves described by Yanai. and Maruyama with forced, easterly, $n=0$ modes is reasonably secure. The existence of an easterly, $n=0$ mode is indeed a result of the beta effect. However, given the forced nature of the observed waves, there is no reason to ascribe an easterly horizontal phase speed, per se, to the beta effect. Indeed both easterly and westerly waves can freely propagate vertically over the equator as internal gravity-type waves. The question may then be asked as to why easterly rather than westerly waves were observed. The answer may be in the recent work of Booker and Bretherton (1967) - where it was shown that at levels where $c=-\omega / k$ $=U$ (i.e., where $\hat{\omega}=0$ ), the wave is almost completely absorbed by the mean flow. Now from figures (2) and (16) in Maruyama (1967) we see that the mean zonal current in the region of observation varied from $-20 \mathrm{~m} / \mathrm{s}$ to $+14 \mathrm{~m} / \mathrm{s}$. Thus, it is not surprising that no waves with these horizontal phase speeds were found. Moreover, the observed phase speed, $-23 \mathrm{~m} / \mathrm{s}$, falls outside this range.

\section{Implications.}

We turn, in this section, to two additional

* Maruyama (1967) assumes, for example, a maximum temperature oscillation of $1^{\circ} \mathrm{C}$ whereas $1.4^{\circ} \mathrm{C}$ is shown in his Fig. 12. Also, filtering reduces amplitudes. Thus, his estimate should be somewhat low. questions: 1) what role do the observed waves play in the quasi-biennial zonal wind oscillation, and 2) what is the nature of the disturbances not isolated in the analysis of Maruyama (1967). These questions are closely related.

In dealing with these questions we must briefly review current thoughts on the momentum budget in the quasi-biennial, equatorial, stratospheric wind oscillation. Models involving eddy diffusion of momentum (Staley, 1963), advection of mean momentum (Lindzen, $1966 \mathrm{~b}$ ), or coriolis torques (Dickinson, 1968) have all been shown to be unlikely (Wallace, 1967, Holton and Wallace, 1968, Holton, 1968). It has been suggested by Tucker (1965), Wallace and Newell (1966) and others that the momentum results from horizontal eddy transfer. This mechanism also appears inadequate. The waves described by Yanai and Maruyama, for example, are such that $\overline{u v} \approx 0$. Other objections are given in Holton and Wallace (1968). We are thus forced to the conclusion that the momentum results from non diffusive, vertical transfer. Now the data used by Yanai and Maruyama were taken in the spring of 1958 when stratospheric westerlies were being replaced by easterlies. Thus a source of easterly momentum is needed. However, as we have seen in section 2, a peculiarity of $n=0$, easterly waves is that they carry westerly momentum.** Their existence, therefore, cannot contribute to the increasing easterlies-and may, in fact, call for a greater source of easterly momentum than would be needed in their absence. As shown in section 2, vertically propagating easterly waves with $n \neq 0$ would, in fact, carry easterly momentum, which, according to Booker and Bretherton (1967) would be absorbed by the mean field at critical levels where $\hat{\omega}=0$. How a spectrum of such internal, gravity-type waves generate a downward propagating quasi-biennial oscillation is shown in a forthcoming paper by Lindzen and

** Note added in proof: In his recent work, Maruyama (1968) reported that westerly monentum is transported upward, at $50 \mathrm{mb}$ level, by the waves of which period are $3-5$ days. 


\section{Holton.*}

If easterly waves with $n \neq 0$ comprise the bulk of the disturbances omitted in Maruyama's analysis, we may ask why they were not clearly seen. One answer is implicit in equ (2). As shown in Lindzen (1967 a), for $n \neq 0$ and doppler shifted periods in excess of a few days, $h$ is on the order of $5 \times 10^{-3} \mathrm{~km}$ or less. Such values of $h$ are associated with vertical wavelengths of less than about $2 \mathrm{~km}$, and wavelengths this small cannot be resolved with the data used by Yanai and Maruyama. It is a matter of some importance that data with sufficient resolution to check this conjecture be obtained.

\section{References}

Booker, J. R. and F. P. Bretherton, 1967: The critical layer for internal gravity waves in a shear flow. J. Fluid Mech., 27, 513-539.

Charney, J. G. and P. G. Drazin, 1961: Propagation of planetary scale disturbances from the lower into the upper atmosphere. J. Geophys. Res., 66, 83-110.

Dickinson, R. E., 1968: On the excitation and propagation of zonal winds in an atmosphere with Newtonian cooling. J. Atmos. Sci., 25, in press.

Eliassen, A. and E. Palm, 1960: On the transfer of energy in stationary mountain waves. Geofy. siske Publ., 22, 1-23.

Haurwitz, B., 1940: The motion of atmospheric disturbances on the spherical earth. J. Mar. Res., 3, 254-267.

Holton, J. R., 1968: A note on the propagation of the biennial oscillation. J. Atmos. Sci., 25, in press.

Holton, J. R. and R. S. Lindzen, 1968: A note on Kelvin waves in the atmosphere. Submitted to Mon. Wea. Rev.

Holton. J. R. and J. M. Wallace, 1968: A diagnostic numerical model of the quasi-biennial oscillation. J. Atmos. Sci., 25, in press.

Lindzen, R. S., 1966 a : On the theory of the diurnal tide. Mon. Wea. Rev., 94, 295-301.

—_ $1966 \mathrm{~b}$ : Radiative and photochemical processes in mesospheric dynamics: Part II, Vertical propagation of long period disturbances near the equator. I. Atmos. Sci., 23, 334-343.

* It should be noted that for odd modes ( $n$ even) like $n=0, \overline{u w}=0$ at the equator. We, therefore, expect even modes will play a greater role than present analyses suggest.
1967 a : Planetary waves on beta-planes. Mon. Wea. Rev., 95, 441-451.

, $1967 \mathrm{~b}$ : Thermally driven diurnal tide in the atmosphere, Quart. J. Roy. Meteor. Soc., 93, $18-42$.

Longuet-Higgins, M. S., 1968: The eigenfunctions of Laplace's tidal equation over a sphere. Phil. Trans. Roy. Soc. London. A 262, 511-607, (No. 1132)

Maruyama, T., 1967: Large scale disturbances in the equatorial lower stratosphere. J. Met. Soc. Japan, 45, 391-408.

Maruyama, T. and M. Yanai, 1967: Evidence of large-scale wave disturbances in the equatorial lower stratosphere. J. Met. Soc. Japan, 45, 196-199.

Maruyama, T., 1968: Vertical transport of momentum due to large-scale disturbances in the equatorial lower stratosphere, to appear in J. Met. Soc. Japan.

Matsuno, T., 1966; Quasi-geostrophic motions in the equatorial area. J. Met. Soc. Japan, 44, 25-43.

Rosenthal, S. L., 1965 ; Some preliminary theoretical considerations of tropospheric wave motions in equatorial latitudes. Mon. Wea. Rev., 93. 605-612.

Rossby, C.-G., 1939: Relation between variations in the intensity of the zonal circulation of the atmosphere and the displacements of the semipermanent centers of action. J. Mar. Res., 2, $38-55$.

Staley, D. O., 1963: A partial theory of the 26month oscillation of the zonal wind in the equatorial stratosphere. J. Atmos. Sci., 20, 506-515.

Tucker, G. B., 1965 : The divergence of horizontal eddy flux of momentum in the lower equatorial stratosphere. Quart. J. Roy. Met. Soc., 91, 356-359.

Wallace, J. M., 1967: A note on the role of radiation in the biennial oscillation. J. Atmos. Sci., 24, 598-599.

Wallace, J. M. and R. E. Newell, 1966: Eddy fluxes and the biennial stratospherir oscillation. Quart. J. Roy. Met. Soc., 92, 481-489.

Wallace, J. M. and V. E. Kousky, 1968: Observational evidence of Kelvin waves in the tropical stratosphere. Submitted to J. Atmos. Sci.

Wilkes, M. V., 1949: Oscillations of the Earth's Atmosphere, Cambridge Univ. Press, Cambridge, $76 \mathrm{pp}$.

Yanai, M. and T. Maruyama, 1966: Stratospheric wave disturbances propagating over the equatorial Pacific. J. Met. Soc. Japan, 44, 291-294. 